\title{
The Complexity of Complex Patients
}

\author{
Monika M. Safford, MD
}

University of Alabama, Birmingham, AL, USA.

J Gen Intern Med 30(12): 1724-5

DOI: $10.1007 / \mathrm{s} 11606-015-3472-6$

(c) Society of General Internal Medicine 2015

A $\mathrm{s}$ the population of the United States ages, and more and more people live with multiple chronic conditions, the issue of complex patients is receiving increasing attention. ${ }^{1,2}$ Physicians have recognized that disease-centric approaches to clinical management have serious limitations for patients with multiple co-occurring medical conditions. This dialogue has been broadened to include multiple sources of complexity, moving well beyond pathophysiology and multiple co-occurring medical conditions, to behavioral and psychosocial sources of complexity that often overshadow physiology as a barrier to achieving optimal health. ${ }^{3,4}$

In this issue of JGIM, Hong, et al. cite their earlier studies demonstrating that this broad conceptualization of complexity resonates with primary care physicians. ${ }^{5,6}$ In those studies, primary care physicians recognized multiple influences on what makes a patient complex, including psychosocial and behavioral domains, not just medical comorbidities. The fact that these doctors considered the 'whole patient' in their conceptualizing of complexity is reassuring and bodes well for primary care.

This broad primary care perspective raises interesting issues for scientists wishing to develop tools to identify complex patients using data extracted from the electronic health record and insurance claims. The novel prediction tool developed by Hong et al. and reported in this issue of JGIM was designed to mimic physician designation of complexity, and it did indeed identify many individuals not identified by more established tools specifically designed to predict utilization - including the Charlson index modified for use in ambulatory care data and a commercial risk prediction tool. ${ }^{7-9}$ An important finding of the study by Hong et al. is that these established tools did very well in predicting emergency department (ED), hospital utilization, and 30-day readmission rates. It is perhaps not surprising that these older tools were not as good at predicting other processes (primary care visits and no-shows) and outcomes of care (cancer screening, risk factor control), for which they were, after all, not designed. The authors rightly conclude that their novel approach may be utilized as a complement to

Published online August 11, 2015 other methods to identify additional at-risk patients, depending on the context.

However, all of the methods evaluated in the report by Hong et al. have important limitations for identifying complex patients. For example, the novel prediction tool demonstrated low sensitivity $(<50 \%)$ for identifying patients with physician-designated complexity. The more established tools in this study were designed primarily to identify high utilizers, but additional endpoints, such as preventive services and risk factor control, are important in primary care. On the other hand, the tool described by Hong et al. missed many patients identified by the more established risk-prediction tools. Each of these approaches, considered alone, may be insufficient to meet the needs of ambulatory population health managers.

One potential strategy to overcome these limitations would be to recognize that one tool is unlikely to suffice for identifying all complex patients. Indeed, especially from the broad perspective of primary care physicians, it quickly becomes clear that interventions will likely differ dramatically depending on which aspect of complexity is the primary driver. For example, a patient who is complex because of multiple endstage conditions, such as co-occurring heart failure and chronic obstructive lung disease, is likely to require very different interventions to preserve health and functioning than one who is complex because of depression. And these patients will require altogether different approaches than a patient who is complex due to homelessness. It therefore follows that we need more work to reliably identify at-risk patients for specific influences along a spectrum of complexity - e.g., psychosocial influences, biological influences, environmental influences - in addition to more generic tools such as the one presented in the study by Hong et al. Of course, in many cases, there will not be a single driver, and a more individualized case management approach may be most effective.

To some extent, health system leaders are already employing strategies to identify and manage complex patients. For example, patients who frequently use the ED are being identified for case management using the simple approach of creating a registry of patients who use the ED frequently. Managers of patient-centered medical homes identify patients who miss appointments as targets for outreach and problemsolving. This straightforward approach is most useful when one particular type of complexity is the main driver of unwanted outcomes - e.g., homelessness and high ED use in winter - and the reason for unwanted outcomes is obvious. 
However, it is clearly preferable to identify 'pivotal problems' that represent upstream opportunity points from which numerous downstream consequences such as high utilization of costly health services follow. Depression is a good example of a 'pivotal problem'. A recent study demonstrated that a peer coaching intervention designed to help diabetic patients with self-management to achieve glycemic control also substantially reduced hospital use in patients with depressive symptoms. ${ }^{10}$ This intervention did not address hospitalization use, but effectively addressing the 'pivotal problem' through frequent supportive telephone contacts created unintended beneficial downstream consequences. Lack of stability in meeting core physiologic requirements such as food, water, or shelter are likely additional 'pivotal problems' that, if addressed, may prevent a host of unwanted downstream consequences. Because of the fragmented nature of our health care system, incentives for understanding key 'pivotal problems' have been limited, but as the enthusiasm and support for population health management grows, so does the opportunity to better understand these upstream nodes.

Indeed, the ideal risk prediction tool would identify individuals well before they experience 'pivotal problems' and subsequently become high utilizers or have uncontrolled risk factors. In addition to research that identifies 'pivotal problems', more work to identify individuals at high near-term risk for experiencing 'pivotal problems' is also needed.

Where does this leave more generic, blanket approaches such as the novel one presented by Hong et al.? It is likely that tools like this (as well as the other two) identify too heterogeneous a group of complex patients to point the way to the immediate action that would improve their situation. Blanket approaches may be more helpful in monitoring progress at the population level, and less so in identifying an at-risk population for specific intervention. From this perspective, Hong et al.'s tool, when used in combination with other risk prediction tools intended to identify individuals at risk for high health care utilization, may be useful for monitoring population health in ambulatory settings with a broader focus that moves beyond ED and hospital use. As such, their paper contributes to the growing body of research intended to provide tools to optimize population health management. The paper also anticipates the move beyond the hospitalization and readmission outcomes that are currently the main focus of many population health managers, providing an approach that is more germane to monitoring population health in the ambulatory setting. From this perspective, it is a welcome and needed contribution to the growing field of population health management.

Acknowledgments: Dr. Safford receives salary support from Amgen for investigator-initiated research on variations in patterns of lipidlowering therapy.

Conflict of interest: Dr. Safford receives funding from NIH, PCORI, Medscape and Amgen for investigator-initiated research.

\section{Sources of funding: K24 HL111154}

Corresponding Author: Monika M. Safford, MD; University of Alabama, Medical Towers 643, 1717 11th Avenue South, Birmingham, AL 35294-4410, USA (e-mail: msafford@uab.edu).

\section{REFERENCES}

1. Boyd CM, Darer J, Boult C, Fried LP, Boult L, Wu AW. Clinical practice guidelines and quality of care for older patients with multiple comorbid diseases: Implications for pay for performance. JAMA. 2005;294:716-724.

2. Tinetti ME, Bogardus ST Jr, Agostini JV. Potential pitfalls of diseasespecific guidelines for patients with multiple conditions. N Engl J Med. 2004;351:2870-2874.

3. Safford MM, Allison JJ, Kiefe CI. Patient complexity: more than comorbidity. The vector model of complexity. J Gen Intern Med. 2007;22(Suppl 3):382-390.

4. Loeb DF, Bayliss EA, Binswanger IA, Candrian C, deGruy FV. Primary care physician perceptions on caring for complex patients with medical and mental illness. J Gen Intern Med. 2012;27:945-952.

5. Grant RW, Wexler DJ, Ashburner JM, Hong CS, Atlas SJ. Characteristics of "complex" patients with type 2 diabetes mellitus according to their primary care physicians. Arch Intern Med. 2012;172:821-823.

6. Grant RW, Ashburner JM, Hong CS, Chang Y, Barry MJ, Atlas SJ. Defining patient complexity from the primary care physician's perspective: a cohort study. Ann Intern Med. 2011;155:797-804.

7. Hong CS, Atlas SJ, Ashburner JM, Chang Y, He W, Ferris TG, Grant RW. Evaluating a model to predict primary care physician-defined complexity in a large academic primary care practice-based research network. J Gen Intern Med. 2015 [3357]. doi:10.1007/s11606-015-3357-8

8. Charlson ME, Charlson RE, Peterson JC, Marinopoulos SS, Briggs WM, Hollenberg JP. The charlson comorbidity index is adapted to predict costs of chronic disease in primary care patients. J Clin Epidemiol. 2008;61:1234-1240.

9. Dang DK, Pont JM, Portnoy MA. Episode treatment groups: an illness classification and episode building system-part i. Med Interface. 1996;9:118-122.

10. Chan JC, Sui Y, Oldenburg B, Zhang Y, Chung HH, Goggins W, Au S, Brown N, Ozaki R, Wong RY, Ko GT, Fisher E, Jade, PP Team. Effects of telephone-based peer support in patients with type 2 diabetes mellitus receiving integrated care: a randomized clinical trial. JAMA Intern Med. 2014; 174:972-981. 